\title{
Numerical and Experimental Studies on Local and Global Instability of Slender System Subjected to a Specific Load
}

\author{
K. SOKÓE* \\ Częstochowa University of Technology, J.H. Dabrowskiego 73, 42-201 Częstochowa, Poland \\ Doi: $10.12693 /$ APhysPolA.138.207 \\ *e-mail: sokol@imipkm.pcz.pl
}

\begin{abstract}
The paper contains the results of theoretical, numerical, as well as experimental investigations on local and global instability of slender system subjected to a specific load. The phenomenon of local and global instability can be found when nonlinear slender systems are studied. The mentioned instability regions are defined during a comparative analysis on the bifurcation load of a geometrically nonlinear structure to a critical load of a corresponding linear one (generally, the linear system is a simplification of a nonlinear one). In the numerical simulation one focuses on an influence of the parameters of the system on vibration frequency and loading capacity on the basis of which the instability regions are plotted. The investigated structure has a defect in the form of a reduced cross-sectional area. The presence of the notch affects both investigated parameters. The studied slender system is loaded by the specific load that leads to the divergence-pseudoflutter shape of the characteristic curve. The differential equations of motion and natural boundary conditions are obtained with the use of the Hamilton principle. The problem is solved with the small parameter method. The main goal of this research is to obtain the global and local instability regions and to decide when to use nonlinear system or to substitute is with the corresponding linear one. Finally, the numerical calculations are compared to the experimental tests.
\end{abstract}

topics: vibrations, stability, piezoceramic, vibration control

\section{Introduction}

The phenomenon of local and global instability can be found when nonlinear slender systems are studied. The mentioned instabilities are defined during a comparative analysis on the bifurcation load of a geometrically nonlinear structure to a critical one of a linear system. Taking into account that a slender system keeps a rectilinear form of static equilibrium, one can find such a magnitude of external load at which the instability occurs. The magnitude of this load is called a bifurcation load for nonlinear systems and critical for linear ones. The local instability takes place when the bifurcation load of a nonlinear system is smaller than the critical load of a corresponding linear one. The global instability phenomenon is present when the bifurcation load of a nonlinear system is greater than the critical one of a corresponding linear structure. The instability point is calculated as a function of the flexural rigidity factor. As presented in [1] at a lower magnitude of the flexural rigidity factor, the local instability occurs. Moreover, an idea of the local and global instability is not reserved to slender systems but it can be also found in studies on sandwich panels [2] and flows [3].

\section{The boundary problem}

The studied column [4] is presented in Fig. 1. The presence of a defect (notch) separates the internal rod into three parts. The definition of natural boundary conditions will satisfy the continuousness of displacements, bending moments, and deflection angles. The column is loaded with

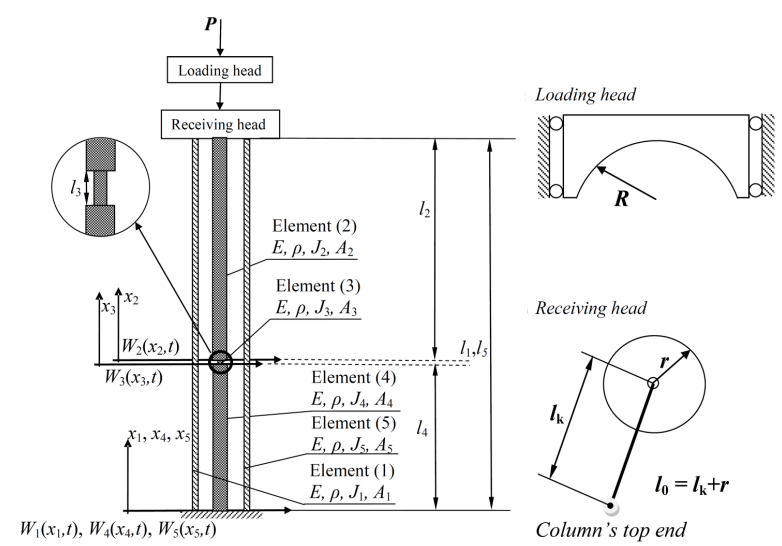

Fig. 1. Investigated system. 
a heads with circular outlines and combined mass $m$. The loading head moves vertically and has a radius $R$. The load receiving head is mounted on the column and has a radius $r$. The length of the elements is defined as $l=l_{1}=l_{5}=l_{2}+l_{3}+l_{4}$. The $l_{0}$ mark shows the length between the contact point of heads and the end of the column.
The Hamilton principle [5, 6] is used during the boundary problem formulation

$$
\delta \int_{t_{1}}^{t_{2}}[T-V] \mathrm{d} t
$$

The energies kinetic $T$ and potential $V$ are defined as:

$$
\begin{aligned}
T= & \frac{1}{2} \sum_{i=1}^{5} \int_{0}^{l_{i}} \rho_{i} A_{i}\left[\frac{\partial W_{i}\left(x_{i}, t\right)}{\partial t}\right]^{2} \mathrm{~d} x_{i}+\frac{1}{2} m\left[\left.\frac{\partial W_{1}\left(x_{1}, t\right)}{\partial t}\right|_{x_{1}=l_{1}}\right]^{2} \\
V= & \frac{1}{2} \sum_{i=1}^{5} \int_{0}^{l_{i}} E_{i} J_{i}\left[\frac{\partial^{2} W_{i}\left(x_{i}, t\right)}{\partial x_{i}^{2}}\right]^{2} \mathrm{~d} x_{i} \\
& +\frac{1}{2} C_{L}\left(\left.\frac{\partial W_{3}\left(x_{3}, t\right)}{\partial x_{3}}\right|_{x_{3}=0}-\left.\frac{\partial W_{2}\left(x_{2}, t\right)}{\partial x_{2}}\right|_{x_{2}=l_{2}}\right)^{2}+\frac{1}{2} C_{H}\left(\left.\frac{\partial W_{3}\left(x_{3}, t\right)}{\partial x_{3}}\right|_{x_{3}=0}-\left.\frac{\partial W_{3}\left(x_{3}, t\right)}{\partial x_{3}}\right|_{x_{3}=l_{3}}\right)^{2} \\
& +\sum_{i=1}^{5} \int_{0}^{l_{i}} E_{i} A_{i}\left[\frac{\partial U_{i}\left(x_{i}, t\right)}{\partial x_{i}}+\frac{1}{2}\left(\frac{\partial W_{i}\left(x_{i}, t\right)}{\partial x_{i}}\right)^{2}\right]^{2} \mathrm{~d} x_{i}+P U_{1}\left(l_{1}, t\right)-\frac{1}{2} P l_{0}\left(\left.\frac{\partial W_{i}\left(x_{i}, t\right)}{\partial x_{i}}\right|^{x_{1}=l_{1}}\right)^{2} \\
& +\frac{1}{2} \operatorname{Pr}\left\{\left[\left.\frac{\partial W_{i}\left(x_{i}, t\right)}{\partial x_{i}}\right|^{x_{1}=l_{1}}\right]^{2}-X^{2}\right\}+\frac{1}{2} P W^{*}
\end{aligned}
$$

$W^{*}=W_{1}\left(l_{1}, t\right)+\left.\frac{\partial W_{1}\left(x_{1}, t\right)}{\partial x_{1}}\right|^{x_{1}=l_{1}}\left(l_{0}-r\right)+r X$

$X=\frac{1}{(R-r)}\left(W_{1}\left(l_{1}, t\right)+\left(l_{0}-r\right)\right.$

$$
\left.-\left.\frac{\partial W_{1}\left(x_{1}, t\right)}{\partial x_{1}}\right|^{x_{1}=l_{1}}\right) \text {. }
$$

The symbols in Eqs. (2) and (3) present: $E$ Young's modulus [GPa], $J_{i}$ - moment of inertia $\left[\mathrm{m}^{4}\right], A_{i}$ - cross-sectional area $\left[\mathrm{m}^{2}\right], \rho$ - material density $\left[\mathrm{kg} / \mathrm{m}^{3}\right], P$ - external load $[\mathrm{N}], U_{i}-$ longitudinal displacements $[\mathrm{m}], W_{i}$ - transversal displacements $[\mathrm{m}], R$ - loading head radius $[\mathrm{m}], r$ load receiving head radius $[\mathrm{m}], m$ mass of heads $[\mathrm{kg}]$, $l_{0}$ - transom length $[\mathrm{m}], C_{L}, C_{H}$ - rotational spring stiffness $[\mathrm{N} \mathrm{m}]$.

The rotational springs are localized in connection of rod 3 with 2 and 4 . Such a creation of the mathematical model will open the possibility of simulation of weakening due to notch presence.

On the basis of (1) one obtains equations of motion, as well as natural boundary conditions which are supplemented by the geometrical ones. The further mathematical operations are done with the small parameter method on the basis of which one presents in this paper results from the first power of the small parameter.

\section{Numerical simulations and experimental tests}

The results of simulations are presented in the nondimensional form:

$$
\begin{aligned}
& p=\frac{P l^{2}}{E J_{1}+E J_{4}+E J_{5}}, \\
& \omega=\sqrt{\Omega^{2} \frac{\left(\rho A_{1}+\rho A_{4}+\rho A_{5}\right) l^{4}}{E J_{1}+E J_{4}+E J_{5}}}, \quad d_{i}=\frac{l_{i}}{l}, \\
& r_{41}=\frac{E J_{4}}{E J_{1}}, \quad r_{34}=\frac{E J_{3}}{E J_{4}}, \quad r_{24}=\frac{E J_{2}}{E J_{4}}, \\
& r_{51}=\frac{E J_{5}}{E J_{1}}, \quad k_{A}=\frac{R}{l}, \quad k_{B}=\frac{r}{R}, \quad k_{C}=\frac{l_{0}}{R}, \\
& c_{L}=\frac{C_{L} l}{E J_{1}+E J_{4}+E J_{5}}, \\
& c_{H}=\frac{C_{H} l}{E J_{1}+E J_{4}+E J_{5}}, \\
& k_{D}=\frac{m}{\left(\rho A_{1}+\rho A_{4}+\rho A_{5}\right) l} .
\end{aligned}
$$

In Fig. 2 the relation between the maximum load of the linear $(\mathrm{KL})$ and nonlinear column at different magnitude of $r_{41}$ can be found.

When $r_{41} \in\left(0, r_{41 L}\right)$ the local instability takes place - the loading capacity of the linear system is greater than the nonlinear one. Points $r_{41 L}$ are marked as black circles. The points localization 


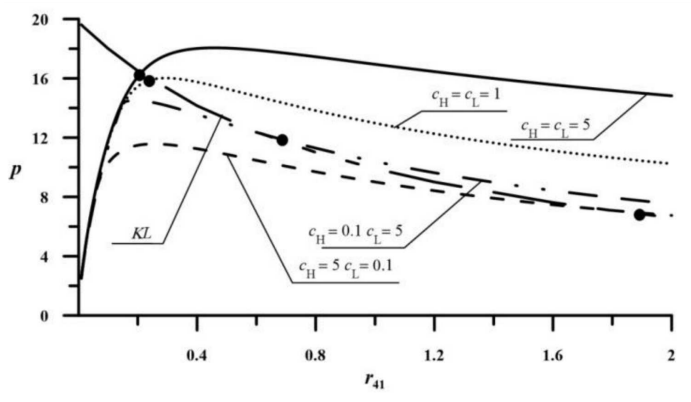

Fig. 2. Local and global instability regions, $k_{A}=0.2, k_{B}=0.2, k_{C}=0.2, r_{24}=r_{51}=1$, $r_{34}=0.578, d_{4}=0.75, d_{3}=0.05$.

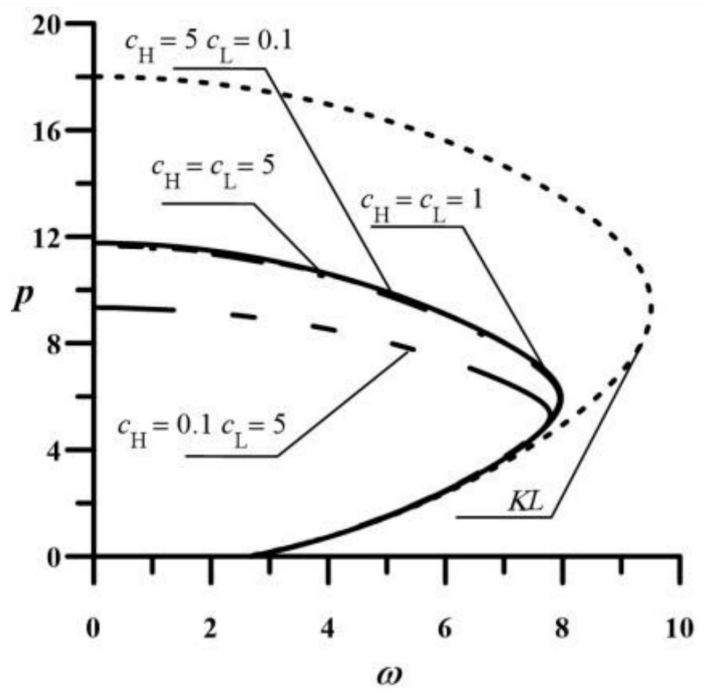

Fig. 3. Local and global instability - characteristic curves, $k_{A}=k_{B}=k_{C}=k_{D}=0.2, r_{41}=0.1$, $r_{24}=r_{51}=1, r_{34}=0.578, d_{4}=0.25, d_{3}=0.05$.

depends inter alia on notch parameters and rotational springs stiffness and others not presented in this publication. At $r_{41} \in\left(r_{41 L}, 2\right)$ the global instability can be seen. It should be stated that the maximum loading of linear column occurs when $r_{41} \approx 0$, while at $c_{H}, c_{L}=\infty$ in the case of nonlinear one.

In Fig. 3 the vibration frequency curves are plotted at a different stiffness of rotational springs.

As presented at $r_{41}=0.1$, one can find that the obtained curves of linear system KL and nonlinear one at the beginning covers each other. An increase in magnitude of external load results in shift of the KL curve in relation to the others and leads to greatest magnitudes of vibration frequency and loading capacity. At every curve one can find a point of maximum vibration frequency above which the change in inclination angle takes place which is characteristic at Tomski's load.

The experimental tests were performed with the following apparatus: accelerometer Bruel\&Kjaer 4508B, the analyzer Bruel\&Kjaer 3560C, PULSE

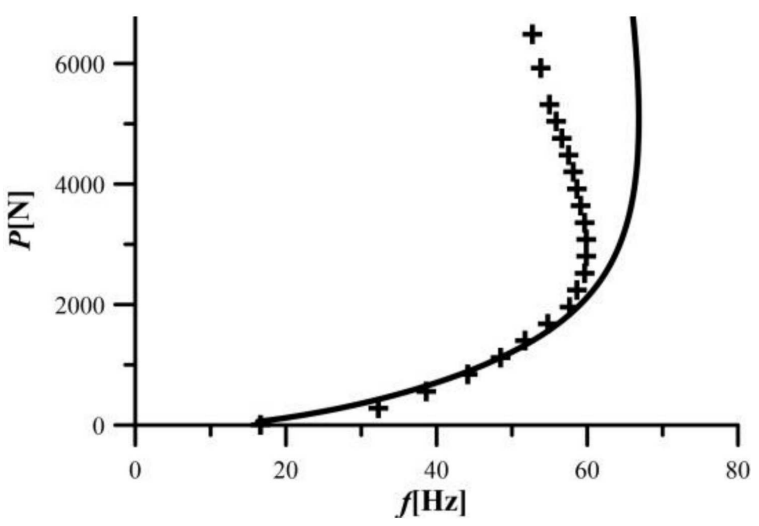

Fig. 4. Theory vs. experiment.

software. The test sample was characterized by $l=0.683 \mathrm{~m}, d_{w}=0.015 \mathrm{~m}, d_{e}=0.012 \mathrm{~m}$, material - aluminum $\left(E=7.5 \times 10^{10} \mathrm{~Pa}\right)$. The notch location $l_{c}=0.17 \mathrm{~m}$ from the support. Notch height/depth $0.002 \mathrm{~m} / 0.004 \mathrm{~m}$. Heads parameters $R=0.04 \mathrm{~m}, r=0.0275 \mathrm{~m}, l_{0}=0.083 \mathrm{~m}$.

In Fig. 4 the continuous line shows the numerical simulation results at $c_{H}, c_{L}=\infty$. Cross-marks show the experimental test data. As plotted at small magnitude of external load the theory and experiment data have great convergence. With an increase in external load the convergence is decreasing which is a result of simplifications in the mathematical model.

\section{Conclusions}

As shown, the control on selection of the crosssectional areas of the elements leads to control of the loading capacity and the vibration frequency which finally may result in change of the complex and expensive nonlinear system into simpler and cheaper linear one.

\section{References}

[1] L. Tomski, S. Uzny, Mechan. Mechanic. Eng. 17, 197 (2013).

[2] J. Pozorska, Z. Pozorski, AIP Conf. Proc. 1738, 480087 (2016).

[3] O. Doarée, D. Langre, J. Fluids Struct. 16, 1 (2002).

[4] S. Uzny, K. Sokół, AIP Conf. Proc. 1648, 850036 (2015).

[5] S. Uzny, K. Sokół, M. Osadnik, Proc 22nd Int. Conf. Engineering Mechanics 2016, Svratka, Czech Republic, 2016, p. 562.

[6] K. Sokół, S. Uzny, in: Proc 22nd Int. Conf. Engineering Mechanics 2016, Svratka, Czech Republic, 2016, p. 514. 